\title{
Erratum to: psychometric evaluation of the Mental Health Continuum-Short Form (MHC-SF) in Chinese adolescents - a methodological study
}

\author{
Cheng Guo ${ }^{1 *}$, Göran Tomson ${ }^{1,2}$, Jizhi Guo ${ }^{3}$, Xiangyun $\mathrm{Li}^{3}$, Christina Keller ${ }^{4}$ and Fredrik Söderqvist ${ }^{5,6}$
}

Unfortunately, the original version of this article [1] contained two mistakes. The text on page 5 reading, "Floor effects were negligible for all items (2\%-14 \%) while substantial ceiling effects were observed except Item 4 (30 \%-40 \%)" should instead have read, "Floor effects were negligible ( $2 \%-14 \%)$ while substantial ceiling effects were observed for all items (30 \%-40 \%) except Item 4".

In Table 5 (Table 1 here), there is a mistake in the ordering of items. The corrected table can be seen below.

\section{Author details}

${ }^{1}$ Medical Management Centre, Department of Learning, Informatics,

Management and Ethics, Karolinska Institutet, Stockholm, Sweden.
${ }^{2}$ Department of Public Health Sciences, Karolinska Institutet, Stockholm, Sweden. ${ }^{3}$ School of Management, Weifang Medical University, Weifang, China. ${ }^{4}$ Jönköping University, International Business School, Jönköping, Sweden. ${ }^{5}$ Center for Clinical Research, Uppsala University, County hospital, Västerås, Sweden. ${ }^{6}$ Competence Center for Health, County Council of Västmanland, Västerås Hospital, Västerås, Sweden.

Received: 28 December 2015 Accepted: 29 December 2015 Published online: 07 January 2016

\section{Reference}

1. Guo C, Tomson G, Guo J, Li X, Keller C, Söderqvist F. Psychometric evaluation of the Mental Health Continuum-Short Form (MHC-SF) in Chinese adolescents - a methodological study. Health Qual Life Outcomes. 2015;13:198. doi:10.1186/s12955-015-0394-2.

Table 1 Distribution in percentage on the lowest and highest rating scale for the items

\begin{tabular}{llcc}
\hline Item & Label & Lowest rating & Highest rating \\
\hline Item 1 & Happiness, joy & $1.5 \%$ & $30.8 \%$ \\
Item 2 & Interested in life & $1.7 \%$ & $34.1 \%$ \\
Item 3 & Content/satisfied & $3.0 \%$ & $30.5 \%$ \\
Item 4 & That you have something important to contribute to the society & $8.9 \%$ & $12.9 \%$ \\
Item 5 & That you belong to a community & $13.9 \%$ & $33.9 \%$ \\
Item 6 & That our society is becoming a better place for all people & $8.2 \%$ & $31.7 \%$ \\
Item 7 & That people are basically good & $5.7 \%$ & $32.6 \%$ \\
Item 8 & That the way society works is logical & $5.3 \%$ & $32.5 \%$ \\
Item 9 & That you like most of your personality & $3.3 \%$ & $3.0 \%$ \\
Item 10 & That you are good at managing responsibility for your daily life & $2.8 \%$ & $36.2 \%$ \\
Item11 & That you have warm and confident relationships with others & $3.6 \%$ & $37.1 \%$ \\
Item12 & That you experience things that will make you grow as a person & $29.4 \%$ \\
Item13 & That you have the confidence to have your own thoughts and that you dare to express them & $4.1 \%$ & $32.1 \%$ \\
Item 14 & That life has a purpose & $2.9 \%$ & $36.9 \%$ \\
\hline
\end{tabular}

\footnotetext{
* Correspondence: guo@ki.se

${ }^{1}$ Medical Management Centre, Department of Learning, Informatics,

Management and Ethics, Karolinska Institutet, Stockholm, Sweden
}

Full list of author information is available at the end of the article 